\title{
UNA REVISIÓN A LOS CONCEPTOS DE COMUNICACIÓN ESTRATÉGICA Y GESTIÓN PÚBLICA EN EL MARCO DE PROCESOS ORGANIZACIONALES PARTICIPATIVOS
}

\author{
A review of the concepts of strategic communication and public \\ management within the framework of participatory organizational processes
}

\author{
Claudia Margarita Mejía ${ }^{1}$, Luis Ricardo Navarro Díaz ${ }^{2}$
}

Recibido: Febrero 24 de 2016/Aceptado: Junio 14 de 2016

\begin{abstract}
RESUMEN
Este artículo analiza procesos de comunicación estratégica en la construcción de identidad, cultura y participación ciudadana, como pilares para asegurar la efectividad de la gestión pública en la búsqueda del cumplimiento de los objetivos y fines de las organizaciones. En este marco, se propone una revisión teórica que aborda el concepto de comunicación estratégica en relación con procesos de desarrollo de la gestión pública de las organizaciones. El impacto del estudio, no solo se encuentra en la posibilidad de ser asumido como referente teórico para la toma de decisiones por parte de los líderes de diversas organizaciones, sino en ofrecer reflexiones y procesos comunicativos pertinentes para realizar procesos de seguimiento y control por parte de los actores sociales pertenecientes a una organización.
\end{abstract}

Palabras clave: Comunicación estratégica, Gestión pública, Organización, Participación.

\begin{abstract}
This article analyzes strategic communication processes in the construction of identity, culture and citizen participation, as pillars to ensure the effectiveness of public management in the search for compliance with the objectives and purposes of organizations. In this context, a theoretical review is proposed to analyze the concept of strategic communication and its relationship with the processes of public management of organizations. The impact of the study, is not only in the possibility of being assumed as a theoretical reference for decision making by the leaders of public organizations, but also to offer relevant reflections from the strategic communication, to execute follow-up processes, observation citizen and public control by social actors belonging to public management.
\end{abstract}

Keywords: Strategic communication, Public management, Organization, Participation.

Cómo referenciar este artículo: Mejía, C. \& Navarro, L. (2016). Una revisión a los conceptos de comunicación estratégica y gestión pública en el marco de procesos organizacionales participativos. Ad-Gnosis, 5(5), 113-126.

\footnotetext{
1. Doctora en Ciencias Gerenciales, Universidad Urbe. Especialista en Gerencia de la Comunicación Organizacional y en Docencia e Investigación Universitaria. Docente investigadora de la Escuela de Comunicación Social y Periodismo de la Universidad Sergio Arboleda, Santa Marta, Colombia. claudiam.mejia@usa.edu.co

2. Doctor en Ciencias Sociales, Universidad del Norte. Docente de la Escuela de Comunicación Social y Periodismo de Universidad Sergio Arboleda, Santa Marta.
} 


\section{Introducción}

El artículo se centra en temas relacionados con la gestión pública y participación ciudadana mediados por procesos de comunicación estratégica. El documento es resultado de un análisis de investigación* que rastrea indicadores constructores de ciudadanía a través del fortalecimiento de una gestión pública que busca la interacción con sujetos-ciudadanos informados, responsables y participativos dentro de un Estado. De acuerdo con el derecho constitucional se define Estado como: agrupación humana, fijada en un territorio determinado en la que existe un orden social, político y jurídico orientado hacia el bien común, establecido y mantenido por una autoridad dotada de poderes de coerción" (Hanauriou, 1980, p.118). Sin embargo se acude a una concepción más amplia y por ello se asume al Estado, como organización económica, política y cultural que se caracteriza por incluir en sus dinámicas, complejos sistemas de interacciones en relación de interdependencia con diferentes actores que intervienen en la dinámica social y en la calidad de vida de sus ciudadanos.

En coherencia con lo anterior, y en conexión con una concepción propia de la comunicación, es pertinente afirmar que diversos autores definen el concepto de Estado como un sistema social relacional. Por ejemplo, Martínez

* El presente artículo es resultado del proyecto de investigación Comunicación Estratégica y Competitividad desarrollado por la línea de Comunicación Estratégica, Cultura y Marca del Grupo de Investigación Comunicación y Sociedad de la Escuela de Comunicación Social y Periodismo de la Universidad Sergio Arboleda, Santa Marta.
(2011), manifiesta que Estado es "Como una unidad política independiente que cuenta con reconocimiento de otros estados. El Estado es una forma de organización social conformada por una red de instituciones que tiene el poder de regular la vida nacional en un territorio determinado, a través de un ordenamiento jurídico y de la soberanía del pueblo" (p.272). Por su parte, Pérez (1999) expresa acerca del Estado que es: "Una institución humana, tiene naturalmente un fin, encierra en su actividad una intención que es la determinante y el motor de toda su estructura el fin será el que determine la competencia material de los diferentes órganos del Estado, y en función de esa competencia se crearan órganos, en este fin esta la razón última del Estado y su diferencia específica con otras sociedades" (p.27). Mientras que Jessop (2003), lo define como "una relación social [...] que puede definirse como un conjunto de instituciones, organizaciones, fuerzas sociales y actividades, incrustadas y reguladas socialmente, seleccionadas de manera estratégica y organizadas alrededor de la toma de decisiones que son vinculantes colectivamente para una comunidad política imaginaria" (p.7).

En este contexto, el ciudadano reconoce a la comunicación estratégica como un factor diferencial en el éxito de la gestión y el relacionamiento entre el sector público y los diferentes sectores de la vida ciudadana, entre ellos, el empresarial, social, académico y la misma sociedad civil. Es así como el estudio propone el diseño de un plan estratégico de comunicación 
que visibilice, responda y articule las acciones que desde los entes públicos se gestionen. El mayor impacto de la propuesta apunta a que los resultados de la investigación, no queden simplemente en un escenario de divulgación académica y exposición mediática, sino trasciendan en la formación de identidad, cultura y participación ciudadana. Ahora bien, la comunicación estratégica se define desde Pérez (2008) como "menos racional y más relacional, menos direccional y más interactiva. La comunicación se hace estratégica en la medida en que sea más proactiva, aprovechando oportunidades, anticipando, administrando y liderando el futuro y sus procesos sociales" (p.111).

La comunicación, pensada estratégicamente, es un factor diferencial y una herramienta que le permite al Estado armonizar, articular y construir tejidos sociales de manera holística, simbiótica y sostenible a largo plazo. En este orden de ideas, la comunicación es al mismo tiempo estrategia y acción, lo cual significa la vinculación entre inteligencia y comunicar con éxito (Costa, 2001). Al respecto, Pérez (2008), afirma que este tipo de comunicación ha abierto, en los tejidos organizacionales contemporáneos, un importante escenario de motivación a partir de la construcción de entretejidos humanos que no solo la fortalecen, sino que además facilitan un ambiente colectivo donde la motivación se comparte de manera simbiótica y grupal.

Sin embargo, estas características no se dan con frecuencia en los entornos de la gestión pública, en gran medida debido a la inmediatez, visión cortoplacista, procesos desarticulados, falta de liderazgo y falta de conocimiento respecto al impacto de la comunicación en los múltiples factores que contribuyen con la construcción de imagen y percepción de la gestión del estado en la opinión pública. De manera contraria se puede plantear que:

Para construir un estado moderno, eficiente y al servicio de la gente es necesario contar con una comunicación estratégica que sea capaz de contribuir a gestar una cultura organizacional de servicio público; que afirme una democracia sustentada en el diálogo con la ciudadanía, legitimando el buen gobierno en la opinión pública; y empodere a la población en el ejercicio de su ciudadanía asumiendo el protagonismo de sus procesos de desarrollo. (Armas, 2013, p.19)

A partir de la cita anterior, es importante identificar al interaccionismo simbólico como eje teórico central de esta construcción teórica, dado que pone al proceso comunicativo como el eje de la vida social, por tanto, todas las relaciones humanas son indefectiblemente relaciones de comunicación, y, por ende, procesos de coproducción e intercambio de sentido (Massoni, 2008), describe la comunicación estratégica como "una metaperspectiva que se ocupa del cambio social conversacional desde los nuevos paradigmas de la ciencia. Rebasa a las teorías y metodologías de la comunicación 
clásica que se restringe al estudio de lo comunicativo y propone abordar la multidimensionalidad de lo comunicacional como encuentro de la diversidad. Entiende a la comunicación como un fenómeno complejo, situacional y fluido" (p.45).

En este sentido, para asegurar una gestión moderna, eficiente y al servicio de la ciudadanía (Navarro, 2016), esta revisión teórica propone como necesidad disponer con procesos de comunicación estratégica que contribuyan a gestar una cultura organizacional orientada a la calidad de la gestión, al diálogo con la ciudadanía, al legítimo buen gobierno y a la construcción de una opinión pública que empodere a la población en el ejercicio de su ciudadanía y su corresponsabilidad en los procesos de desarrollo. Al respecto, Pérez (2008), retoma la idea que asocia la interacción simbólica con procesos de evolución de la interacción físico-química, ya que en la medida en que el hombre avanza en los niveles culturales tiende a "sustituir los actos ejecutivos por actos expresivos, es decir, la fuerza por la interacción simbólica, a través de actos comunicativos que le permiten consensuar discrepancias y resolver conflictos con una menor pérdida de energía" (Serrano, 1981, citado por Pérez, 2008, p.430).

Por consiguiente, son los procesos de comunicación gestores vitales de las tendencias organizacionales de la sociedad actual, vinculadas estas con nuevas formas de organización ciudadana cuya finalidad es contribuir, desde todos los estamentos, en el desarrollo social, económico, cultural y político hacia el mejoramiento de los indicadores de la calidad de vida de sus habitantes. Ahora bien, el acceso a mediaciones tecnológicas relacionadas con la sociedad de la información y la comunicación, han acelerado estas modalidades de asociación, cooperación e integración, propiciando un mayor acceso, rapidez y globalidad de la información. La gestión pública soportada en procesos de comunicación estratégica mediados por la tecnología, agilizan el intercambio de opiniones y propuestas por parte de los ciudadanos, fortalece los procesos para el desarrollo social y vincula objetivos gubernamentales y políticas públicas con actores y organizaciones y entornos culturales, políticos y económicos.

Para construir un Estado moderno, eficiente y al servicio de la gente es necesario contar con una comunicación estratégica que sea capaz de contribuir a gestar una cultura organizacional de servicio público; que afirme una democracia sustentada en el diálogo con la ciudadanía legitimando el buen gobierno en la opinión pública; y empodere a la población en el ejercicio de su ciudadanía asumiendo el protagonismo de sus procesos de desarrollo. (Armas, 2013, p.17)

En coherencia con lo anterior, y en contextos de la gestión pública contemporánea, los procesos de comunicación son valorados a nivel estratégico. De esta forma, la efectividad de 
los contenidos a comunicar, ocupa cada vez más tiempo en las discusiones de la alta dirección de las organizaciones. Las decisiones estratégicas tanto en las organizaciones privadas como públicas tienen su punto de partida en el cómo repensar la forma en que se debe comunicar los asuntos de interés público.

Por tanto, la comunicación en la gestión pública constituye uno de los principales indicadores de aceptación y desarrollo de los gobiernos locales; de tal manera que se convierte en el epicentro de la visión colectiva de las políticas públicas, dando mayores posibilidades de acercamiento o aproximación a las respuestas sociales de problemáticas ciudadanas. Ante ello, se hace pertinente pensar desde los procesos de comunicación estratégica vinculados al desarrollo de la gestión pública, la implementación de espacios de socialización, reconocimiento y discusión de los procesos, que bien pueden ser sistematizados a través de centros de seguimiento o de observación de medición ciudadana que promuevan escenarios de análisis y propuestas ciudadanas desde la sociedad civil.

\section{Referentes teóricos}

\section{La transformación del concepto de gestión}

Desde los modelos tradicionales, la concepción del estado se asocia con la figura de un ente regulador y prestador de los servicios públicos, protector de los intereses y las necesidades básicas de una sociedad mediante procesos de gestión pública. Zafra (1990) define este término como:
Grupo territorial duradero, radicalmente comunitario, estrictamente delimitado, moderadamente soberano frente a otros, que se manifiesta como máximamente comprensivo en el plano temporal y en cuyo seno, sobre una población, con creciente homogeneidad y sentido de autopertenencia, una organización institucional eminentemente burocrática, coherente y jerarquizada, que desarrolla una compleja gobernación guiada conjuntamente por las ideas de seguridad y prosperidad. (p.74)

En relación con lo anterior, cabe resaltar que parte de la función del Estado consiste en afirmar y hacer respetar el orden y equilibrio social (Bergeron, 1993). En algunas ocasiones, esta gestión se ha desarrollado a través de modelos autoritarios o paternalistas-centralizados, cuyo objetivo principal se ha enfocado en la oferta de servicios sociales y públicos unidireccionales en los que no es tenido en cuenta el ciudadano como beneficiario de estos servicios. Según Oyarce (2011), este modelo denominado administración burocrática se caracteriza por poseer un trabajo administrativo lineal, mecanizado y fragmentado (división del trabajo), así como, una estructura jerárquica rígida, una orientación al cumplimiento del mandato público (leyes, decretos, funciones del estado), con toma de decisiones centralizadas y con dirección autócrata.

Sin embargo, la sociedad ha cambiado y con ella sus ciudadanos, quienes ya no solo desean 
la satisfacción de sus necesidades e intereses, sino que quieren un nuevo modelo de gestión, mucho más participativa, dialogante, incluyente, que tenga en cuenta sus ideas y propuestas ya desde el ámbito de usuario o beneficiario de los servicios y actividades públicas. Es allí donde empiezan a emerger nuevos paradigmas y modelos que aseguren el cumplimiento de los objetivos a corto, mediano y largo plazo. En gerencia pública, caracterizada por la orientación a la gestión, la productividad y la eficiencia bajo un modelo donde los funcionarios son gestores públicos, los mandatos pasan a ser logros alcanzados a través de metas, y una actuación fundamentada en la transparencia, flexibilidad, descentralización y autonomía, orientación al servicio, imagen de confianza y credibilidad (Henríquez, 2013).

Desde este mismo paradigma se desprende el concepto de public governece, cuyas principales características se enfocan a procesos participativos, regulación articulada de todos los actores, sistemas de servicios eficientes y eficaces, desempeño basado en la motivación, competitividad, meritocracia, remuneración justa y compromiso laboral. Al respecto, Natera (2004), plantea que gobernanza se refiere a un nuevo estilo de gobierno, diferente de la estructura y control jerárquico, del mercado, caracterizándose por un nivel mayor de cooperación entre Estado y actores no estatales. Este tipo de gobernanza se encuentra relacionada con lo que el Movimiento América Europa de Regiones y Ciudades-AERYC (2005), definió en su reunión anual como "un nuevo arte de gobernar los territorios, cuyo objeto es la capacidad de organización y acción de la sociedad, su medio es la gestión relacional o de redes, y su finalidad es el desarrollo humano" (párr. 2).

De forma articulada con el concepto propuesto en el párrafo anterior, es pertinente identificar también el nuevo paradigma de la gerencia estratégica de la gestión pública para la creación de valor público. A través de este modelo se asigna gran importancia a los procesos de interacción entre organizaciones responsables y ciudadanos/comunidades, a las dinámicas de retroalimentación y percepción mediante diálogos para repensar los procesos de las organizaciones que tributen a valorar las acciones en términos de servicios, resultados, confianza y legitimidad. En este paradigma se centra el objetivo principal de la gestión pública, es decir, de lo que se trata es de generar valor público desde la respuesta a problemas relevantes para los ciudadanos, incluyendo nuevas oportunidades, procesos de construcción de comunidad, ciudadanía, democracia y capital social. Es así como la comunicación pública se define como una herramienta poderosa en manos de los equipos de gobierno, muy importante para explicar, justificar y, en consecuencia, legitimar las decisiones políticas asumidas en cada periodo legislativo. "La comunicación pública: no consiste simplemente en informar, sino que debe servir como instrumento para asegurar la participación de los ciudadanos en la vida pública y hacerles responsables" (Campillo, 2010, p.18). 
En este orden de ideas, los procesos de comunicación estratégica incluyen acciones hacia el aseguramiento de marcos de eficacia, eficiencia, equidad y sostenibilidad por parte de las organizaciones, siempre teniendo en cuenta dimensiones relacionadas con la perspectiva ética de los sujetos, asociada con aspectos como responsabilidad, solidaridad, compromiso y equidad. De esta manera, se asumen las organizaciones como sistemas complejos de interrelación múltiple con otras organizaciones. En este contexto, la gestión pública implica acciones asociadas con la construcción de ciudadanía definidas desde la posibilidad de comunicar los planes de acción de los sujetos, la construcción de soluciones acordes con las necesidades y expectativas de la ciudadanía, el fomento de la participación y la co-responsabilidad de la sociedad civil en las decisiones relacionadas con los asuntos públicos, la coordinación de recursos efectiva y eficientemente para producir el valor esperado, la posibilidad de la innovación y el empoderamiento.

La comunicación, estrategia de la gestión pública moderna

Según Pérez (2008), la comunicación, pensada en relación con ámbitos de la gestión y de la administración, implica un componente estratégico para gestionar mensajes y significados, siempre en búsqueda de construir procesos de interacción y relacionamiento con los diferentes grupos de interés. Es así como la dimensión comunicativa de cualquier organización es posibilitadora de procesos de participación y por ende de fortalecimiento de los tejidos socia- les, dada su influencia en los asuntos públicos y su respectiva gestión, así como su incidencia en el desarrollo de las comunidades.

Este estudio apuesta por la concepción de una comunicación estratégica, entendida no solo desde el intercambio de mensajes por parte de los actores sociales, sino más bien desde la interacción de actores con intereses diversos en contextos en los cuales, unos buscan persuadir a otros a partir de la planificación previa de los contenidos de los mensajes que se van a intercambiar y de los medios que van a utilizarse (Pérez \& Massoni, 2009). El análisis y la gestión de los asuntos públicos se orientan hacia el reconocimiento de consensos y disensos de todos los sectores de la sociedad respecto a las condiciones de decisión y acciones favorables a los intereses colectivos, es decir, la gestión pública busca consensuar entre los múltiples intereses particulares para diseñar políticas, acciones y escenarios participativos. Sin embargo, la diferencia de posiciones es un elemento ineludible dentro de cualquier organización. Esta tesis se puede sustentar desde Elizalde (2009):

La perspectiva comunicativa se puede definir en tres principios de la gestión pública: es un mecanismo de influencia importante, es un agente transformador, es una herramienta de resolución de conflictos y problemas para mejorar las condiciones de la vida democrática, por tanto la gestión de asuntos públicos debe considerar esta variable para la 
obtención de consenso o la producción de disensos públicos. (p.25)

En contraste con Elizalde, este estudio asume la comunicación de forma distante a la categoría herramienta, y más bien la propone como un proceso de producción de sentidos. De forma precisa pensar en una comunicación transformadora y vinculante con consensos y disensos, hace parte de la construcción de la idea principal que se pretende demostrar en este texto. Cabe anotar que la gestión de los temas con representatividad, en la comunidad, necesitan de ciertas perspectivas, especialmente locales, que permitan observar y repensar las diferentes decisiones y soluciones a los problemas más frecuentes, sobre todo para identificar la perspectiva de los actores sociales involucrados de forma activa en los asuntos públicos.

Por otra parte, si la comunicación es considerada como un proceso de producción de sentidos y, por consiguiente de resolución de problemas emergentes en las y entre las organizaciones, es necesario redefinir la gestión de los asuntos públicos sin excluir los procesos propiamente democráticos relacionados con la búsqueda del entendimiento o el reconocimiento de las diferencias.

Desde lo anterior, se hace necesario identificar modelos teóricos de la comunicación pertinentes para sustentar la vinculación entre comunicación estratégica y la gestión pública, conceptos centrales en esta publicación. Una breve revisión de esos modelos obliga a revisar los planteados a mediados del siglo XX, centrados en estructuras de transmisión $y$ medición de información, tales como los propuestos por la teoría matemática de la información. En este caso, es importante subrayar que el énfasis de este modelo teórico radicaba solo en la transmisión efectiva del mensaje; luego se integraron las propuestas, funcionalistas, estructuralistas y los estudios relacionados con la comunicación como un proceso cultural, así como los pensamientos de la línea teórica crítica de la comunicación, asociados a dinámicas propias de la razón dialógica y la emancipación.

La comunicación estratégica integra varias de estas dimensiones teóricas y se convierte en una apuesta de comunicación integral, holística y participativa que puede ser asumida desde y para diversos tipos de organizaciones. En el caso de organizaciones relacionadas con la gestión pública, la comunicación estratégica propone un giro teórico el cual apunta a desplazar el modelo desde estructuras propias de propuestas tradicionales de transmisión de información, unidireccional, fragmentada a posibilidades comunicativas pensadas desde las relacionales, la participación y la construcción compleja de los sentidos de la organización. Al respecto, Rodríguez y Opazo (2008), señalan que "se puede entender una organización desde las perspectivas de las redes comunicativas que constituye su columna vertebral y permiten la coordinación de los esfuerzos de los miembros tras el logro de los objetivos definidos para su gestión" (p.28). 
Ahora bien, uno de los elementos más importantes de un proceso de comunicación estratégica puesto en marcha en contextos de la gestión pública, es la comunicación interna. Esta es representada comúnmente por los miembros de una organización o empresa como eje fundamental en el servicio de una gestión administrativa moderna e innovadora.

La comunicación interna, correctamente entendida, es la gran herramienta y a la vez el contenido último de los estilos de dirección democráticos, que caracterizan la gestión pública contemporánea (...) La comunicación interna no es un fin, sino un medio y una herramienta insustituible para desarrollar las nuevas competencias, entre ellas: la flexibilidad, la polivalencia, la apertura a cambios, el espíritu de la participación, el talante innovador, el trabajo en equipo. (García, 1998, p.10)

El mismo autor realiza una interesante disertación (1998) sobre los dos escenarios que convocan y dan vigor, comúnmente, al desarrollo de una comunicación interna en las organizaciones modernas, el autor los identifica como los acontecimientos de tarea y de relación humana. Se entiende por acontecimientos de tarea: como una actividad del proceso del "know how" de actividades ritualizadas a este dominio que pertenecen a las comunicaciones internas rutinarias. Por su parte, los acontecimientos de relación humana comprenden el desarrollo de un contenido preferentemente enfocado a las ideas, las actitudes, creencias y valores que refuerzan una cultura. A este dominio pertenecen las comunicaciones no rutinarias que dan lugar a las noticias.

Teniendo en cuenta que la necesidad de las organizaciones públicas se enmarca, entre otros aspectos, en la competitividad y relacionamiento de los actores, el concepto de estrategia se propone como central para pensar procesos de comunicación relacionados con dinámicas de gestión pública. En la actualidad el término estrategia se emplea con tal facilidad y asiduidad que casi toda gestión en procura de un objetivo puede ser estratégico. Preciado y Guzmán (2011), afirman al respecto que:

La comunicación estratégica ha sido vista como un modelo que representa numerosos beneficios cuando se traslada al campo de las organizaciones públicas, por varias razones: a) Posibilita que el gobierno trabaje y sea visto como un sistema, como una totalidad, o bien como una unidad hecha de partes; $b$ ) Se aborda desde una visión en la que la organización pública es analizada desde la relación con su entorno y no como un ente aislado al que debe servir; c) Evita caer en miradas cortoplacistas; y d) Permite mostrar la articulación en las políticas, porque al integrar sus acciones en un ciclo más largo, el gobierno enuncia su discurso en torno de ideas y los ciudadanos toman conciencia acerca de la 
manera como este constituye su rumbo. (pp.57-58)

Es así como se hace referencia a términos como publicidad estratégica, mercadeo estratégico, negociación estratégica, planeación estratégica y comunicación estratégica, entre otros. Se puede caracterizar, entonces, la comunicación estratégica como una forma de actuación y participación que asume todo sujeto al entrar en relación con los demás. Se trata de poner en escena las pretensiones de los sujetos y las organizaciones, e intentar establecer acuerdos o reconocer diferencias dentro de la estructura organizacional. En todo contexto, un proceso de comunicación estratégica requiere trazar una metodología, orientar unas acciones en virtud de objetivos propuestos y verificar los resultados en contraste con lo planeado. Tal como lo aseguran Preciado y Guzman (2011):

Las condiciones para que la comunicación de una entidad sea estratégica son las siguientes: a) La comunicación debe considerar elementos central en la toma de decisiones... deben participar de forma activa en el diseño de la estrategia corporativa; b) Los objetivos de la estrategia deben guardar consonancia con los de la estrategia global... c) En el diseño de la estrategia de comunicación se contemplan tres niveles que articulan lo general con lo específico: el estratégico o global, el táctico o intermedio y el operativo. (pp.52-53)
La anterior apuesta, subraya la necesidad no solo de analizar el beneficio que para una organización tiene desarrollar una comunicación estratégica que le permita dialogar con sus sujetos, sino la oportunidad de lograr el cumplimiento de los intereses de la organización a través de una propuesta de comunicación que agrega valor estratégico a la gestión.

La comunicación estratégica sirve al hombre de acción para alcanzar sus metas en sociedad, ayudándose por medio de la interacción simbólica a que los resultados finales de sus actividades sean económicas, políticas y sociales, estén lo más cercanos posibles a sus objetivos, implica administrar la herramienta (controlando y organizando anticipadamente el poder de la comunicación), pero sobre todo gestionar el futuro (actuando ahora) para así lograr, por medio de la comunicación, una mejor coordinación con los otros actores. (Pérez, 2008, p.459)

No obstante, la efectividad en la comunicación para el alcance de los objetivos corporativos, tanto el ámbito privado como público, deben ser evaluados, observados y repensados con miras a su perfeccionamiento en el ámbito social. De ahí, tal como se propone en párrafos anteriores, la pertinencia de los observatorios de comunicación en la actualidad. En síntesis, el entorno actual de las organizaciones y su impulso a la consecución permanente de indi- 
cadores, la evidente competitividad y exigencias que determinan la gestión empresarial a nivel local e internacional, incitan a fomentar desde la académica, escenarios donde las empresas y unidades productivas locales puedan encontrar apoyo en la utilización de la comunicación como un insumo estratégico.

\section{Resultados}

Los procesos de comunicación estratégicos diseñados a partir de contenidos basados en los indicadores de percepción ciudadana y calidad de vida, es considerado como oportunidad para identificar debilidades y oportunidades que tiene la ciudadanía en relación con la gestión pública, ya que a partir de una estrategia participativa es posible construir mensajes hacia la gestión correctiva y de mejoramiento.

En coherencia con lo anterior, los resultados expuestos se convierten en pertinentes y referencia para la toma de decisiones en la ciudad. Sin embargo, el impacto de los resultados no puede quedarse solo en mostrar una radiografía de la organización, sino que debe conducir a la elaboración de una estrategia de gestión pública que construya cultura ciudadana participativa y que genere co-responsabilidad ciudadana. Las reflexiones teóricas aquí desarrolladas apuntan a que los procesos de gestión de los asuntos públicos se articulen, en cuanto a la toma de decisiones y acciones públicas, a dinámicas de comunicación estratégica.

Tello, Bastidas y Ramos (2009), confirman lo anterior al exponer que "la gestión pública está configurada por los espacios institucionales y los procesos a través de los cuáles el Estado diseña e implementa políticas, suministra bienes y servicios y aplica regulaciones con el objeto de dar curso a sus funciones" (p.16). Es por ello, que su exposición puede incidir tanto en la agenda de los gobiernos como en la construcción de la opinión pública, lo que influye en los procesos de percepción ciudadana sobre la gestión de la organización o en este caso del estado. En este sentido se recomienda la confección de estrategias de comunicación que superen las propuestas de los modelos tradicionales de transmisión de información y trasciendan la mera divulgación. Es así como los indicadores de gestión pública se identifican como una oportunidad para construir interlocución entre la administración y la ciudadanía, de tal manera que se fortalezcan los canales empleados para desarrollar los proceso comunicativos, se incentiven las dinámicas participativas de los sujetos involucrados y se facilite el diálogo con la entidad pública que genera la gestión.

De esta manera, la comunicación desarrolla unas acciones estratégicas que permiten monitorear la gestión pública, evidenciar el nivel de participación ciudadana y la corresponsabilidad en las acciones estratégicas a partir de estos indicadores.

De igual forma, se destaca que la socialización de los resultados de la gestión pública, per- 
mite a los líderes de la organización diagnosticar el estado de las acciones de los sujetos $y$, con base en ello, decidir planes de acción que tributen en la consecución de los indicadores propuestos. Por lo tanto, los indicadores de gestión pública pueden servir de insumo para establecer un plan estratégico de comunicación, que sea mucho más que un mapa de divulgación y exposición a la prensa, para convertirse en un documento abierto que refleje el nivel de comunicación entre el sector público y la sociedad en general. Las mesas abiertas de trabajo, los procesos de diálogo y debate y el uso de las redes sociales son tres mecanismos pertinentes para estos fines.

\section{Conclusión}

En síntesis, se evidencia la necesidad cada vez más crítica de desarrollar acciones orientadas hacia la implementación de una comunicación mucho más estratégica en el sector público, cohesionadas con las decisiones directivas y del más alto nivel, de tal manera que logre articular todos los actores de la esfera social para lograr el cumplimiento colectivo de los objetivos institucionales, políticos, sociales y económicos, delimitados de manera conjunta por dichos actores.

Se propone por tanto, construir en las organizaciones públicas una cultura de gestión organizacional fundamentada en una comunicación estratégica incluyente, visionaria, participativa y activadora de mecanismos de cooperación, participación y corresponsabilidad ciudadana, junto con todos los actores que intervienen en los procesos de desarrollo, competitividad y calidad de vida de sus habitantes.

La comunicación estratégica se convierte así en un espacio de mediación de los procesos y políticas públicos para establecer planes de mejoramiento hacia el éxito y cooperación participativa no solo para el logro de las metas, sino más importante aún para la construcción de una cultura ciudadana participativa, consciente y corresponsable de la gestión pública.

Se trata de proponer una comunicación estratégica que aporte en la construcción de redes y dinámicas eficiente en el ejercicio de la gestión pública de manera incluyente, con el fin de fortalecer las relaciones entre los actores sociales implicados, afianzar la imagen de los líderes y funcionarios públicos; así como la confianza entre los sujetos sociales, a través estrategias, medios y canales contextualizados en la realidad social y del entorno público de dicha gestión.

La planificación, seguimiento y mejoramiento continuo de los procesos comunicativos de la gestión, se fundamenta en la tesis de definir como pertinente implementar el modelo de comunicación estratégica en gestión de las organizaciones y entidades públicas, reconociendo el significativo impacto de su quehacer en los seres humanos que dependen de ella. 


\section{Referencias}

AERYC (2005). Regiones y ciudades ante el desarrollo humano contemporáneo: La gobernanza democrática. En Memorias reunión anual Movimiento América Europa de Regiones y Ciudades. Valencia: Dirección General de Administración Local. Junta de Andalucía. Disponible en: http://www.juntadeandalucia.es/ export/drupaljda/ade050907_v3_definitiva.pdf

Armas, S. (2013). Desafíos de la comunicación estratégica en la gestión pública. En Hablemos, más voces para la comunicación. Lima: Ocina General de Comunicación Social de la Presidencia del Consejo de Ministros.

Bergeron, G. (1993). El estado en funcionamiento en fonctionnement. Paris: Ed. L'Harmattan.

Campillo, A. (2010). Comunicación pública y administración municipal. Una propuesta de modelo estructural. Revista Pensar la Publicidad, 4, 45-62.

Costa, P. (2001). Comunicación Pública en el ámbito local. En Benavides, J. (2001), Dirección de comunicación empresarial e institucional. Barcelona: Gestión.

Elizalde, L. (2009). Gestión de la comunicación pública. Barcelona: Bosch.

García, J. (1998). La Comunicación interna. Madrid: Ediciones Díaz de Santos.

Hanauriou, A. (1980). Derecho Constitucionale instituciones políticas. Barcelona: Ariel.
Henríquez Fuentes, G. R. (2013). Investigación cualitativa en modelos de gestión logística y sus estrategias de desarrollo en la costa Caribe colombiana. Ad-Gnosis, 2(2), 65-85.

Jessop, R. (2003). El futuro del estado capitalista. Cambrige: Polity Press.

Martínez, M. (2011). Redes alternativas de comunicación, framing y la construcción del poder político. Revista de Ciencias Sociales, 6, 269-291.

Massoni, S. (2008). Estrategias. Los desafios de la comunicación en un mundo fluido. Rosario: Homo Sapiens.

Massoni, S. (2008). Los desafíos de la comunicación en un mundo fluido. Revista FISEC-Estrategias, 10, 45-56. Disponible en: http://www.cienciared.com.ar/ ra/usr/9/254/fisec_estrategias_n10_ pp45_56.pdf

Oyarce, H. (2011). Gerencia pública. Santiago de Chile: Instituto Latinoamericano y del Caribe de Planificación Económica y Social (ILPES). Disponible en: http:// www.cepal.org/ilpes/noticias/paginas/4/45114/Gerencia_Publica2011_ HOYARC

Natera, A. (2004). La noción de gobernanza como gestión pública participativa y reticular. Madrid: Universidad Carlos III de Madrid.

Navarro, L. (2016). Entre esferas públicas y ciudadanías. Barranquilla: Ediciones Uninorte.

Pérez, R. (2008). Estrategias de Comunicación. Barcelona: Ariel. 
Pérez, F. (1999). Teoría del Estado. México: Po- $\quad$ Rodríguez, D. \& Opazo, M. (2008). Comunicarrúa.

Pérez, R. \& Massoni, S. (2009). Hacia una teoría general de la estrategia. El cambio de paradigma en el comportamiento humano, la sociedad las instituciones. Barcelona: Ariel. ciones de la organización. México: Alfaomega.

Tello, P., Bartidas, D. \& Ramos, J. (2009). Estado y Gestión Pública. México: Instituto para la Democracia y la Asistencia Electoral (IDEA).

Preciado, A. \& Guzmán, H. (2011). Usos y prácZafra, J. (1990). Teoría Fundamental del Estaticas de comunicación estratégica en do. Pamplona: Universidad de Navarra. organizaciones públicas y políticas. $R e$ vista Folios, 49-73. 